\title{
Sequencing Skippy: the genome sequence of an Australian kangaroo, Macropus eugenii
}

\author{
Elizabeth P Murchison and David J Adams* \\ See related research by Renfree et al., http://genomebiology.com/2011/12/8/R81
}

\begin{abstract}
Sequencing of the tammar wallaby (Macropus eugenii) reveals insights into genome evolution, and mammalian reproduction and development.
\end{abstract}

Keywords Marsupial, genome, kangaroo, wallaby

The kangaroo is an iconic and much loved Australian. To Australians it is an animal that symbolizes our individuality and vigor, and its unique hopping gait is famous around the world. The kangaroo is a marsupial, a mammal with a short gestation period and a prolonged lactation period, which usually occurs inside a specialized pouch. Marsupials diverged from the eutherian lineage some 130 to 148 million years ago [1,2], and today marsupials are found in Australia, New Guinea and the Americas. However, kangaroos are found only in Australia, where they have adapted to the harsh, arid environment. Kangaroos are members of the Macropodidae family (macropod, meaning 'large foot'), which includes four species that are commonly referred to as kangaroos as well as a number of smaller macropodids, including wallabies, tree-kangaroos, quokkas and pademelons. The tammar wallaby (Macropus eugenii) is one of the smaller wallabies, and is found on islands off the coasts of South and Western Australia (Figure 1). This diminutive member of the kangaroo family is widely used in developmental, reproductive and physiological studies and is a favorite at zoos worldwide. An article in this issue of Genome Biology reveals the genome sequence of the tammar wallaby, providing unique insights into the evolution of marsupial and mammalian genomes [3].

*Correspondence: da1@sanger.ac.uk

Wellcome Trust Sanger Institute, Wellcome Trust Genome Campus, Hinxton, Cambridge CB10 1SA, UK

\section{Sequencing the tammar genome}

Despite the importance of marsupials in the mammalian evolutionary tree, until recently only one marsupial genome had been completely sequenced: the genome of the grey short-tailed opossum Monodelphis domestica [4]. The interesting biology of the macropodid family, including their specialized body plan, prolonged lactation, and their ability to delay the development of their young (embryonic diapause), made them an attractive choice for whole-genome sequencing. As a smaller member of the kangaroo family that breeds well in captivity and is easy to handle, the tammar wallaby was the obvious macropodid species to sequence.

Like the tammar itself, the researchers who sequenced this kangaroo genome were highly adaptable. They employed an arsenal of technologies to sequence the tammar genome, including capillary whole-genome shotgun sequencing, ABI-SOLID, Roche/454 and Illumina sequencing. Integration of these data with a physical map and linkage map produced an assembly with an N50 scaffold size of $34.3 \mathrm{~kb}$ in 324,751 scaffolds, a subset of which were mapped and ordered onto superscaffolds corresponding to chromosomes.

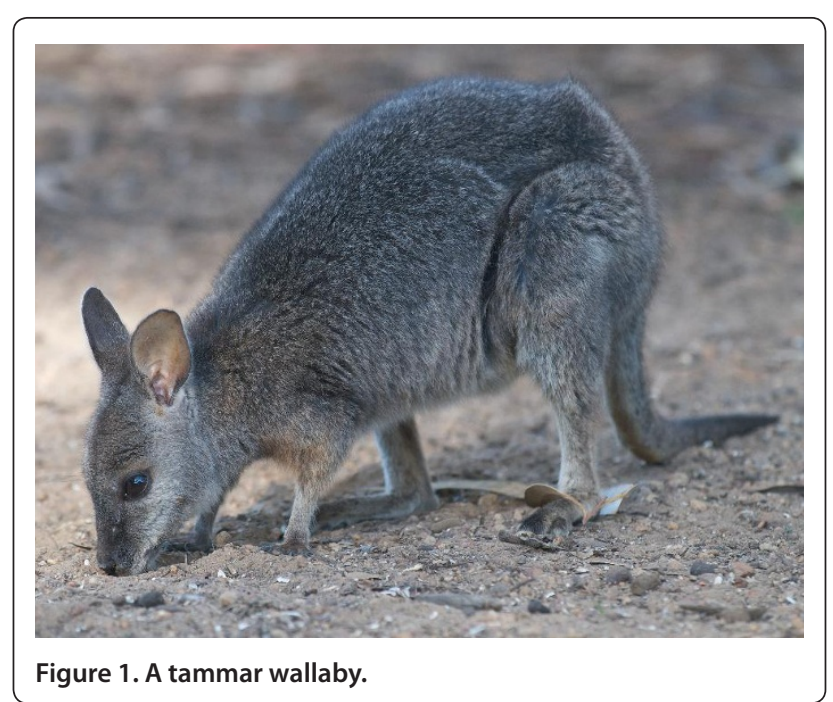


Interestingly, the tammar was found to have greatly reduced centromere sizes compared with human and mouse [5], resulting in a compact genome, estimated to be $2.9 \mathrm{~Gb}$ in size.

\section{Sex and the $X$}

Although marsupial sex chromosomes share common ancestors with their eutherian cousins, the marsupial $\mathrm{X}$ and $\mathrm{Y}$ chromosomes are considerably smaller in size than those of humans and mice. The $\mathrm{X}$ chromosomes of humans and wallabies share many of the same genes, albeit in a different order. However, one notable gene that is missing on the wallaby $\mathrm{X}$ chromosome is XIST [6], the non-coding RNA gene responsible for $\mathrm{X}$ chromosome inactivation in eutherian mammals. Although dosage compensation does occur in female wallabies, it is incomplete and always involves the shut down of the paternally inherited copy of the $\mathrm{X}$ chromosome. Interestingly, the tammar $\mathrm{X}$ chromosome seems to harbor a large number of conserved non-coding regions, perhaps providing clues as to the mechanism of $\mathrm{X}$ inactivation in marsupials.

Orthologs of the genes essential for gonadal differentiation, spermatogenesis and development are all largely conserved between the tammar and other mammals. This suggests that despite divergent evolution and strikingly different embryonic morphology, the basic machinery of reproduction and development has been conserved in the tammar.

\section{Small and mighty}

The wallaby genome offers some surprises in its repertoire of small RNAs. Similar to other mammalian genomes, the wallaby has a set of miRNAs and piRNAs. Some of the miRNAs are conserved with other mammals; however, most of the miRNAs identified in the tammar wallaby are novel. These include new miRNAs in the $H O X$ cluster. In addition, the authors made the unexpected discovery of a new class of small RNAs, which they named centromere repeat associated short interacting RNAs (crasiRNAs) [5]. These RNAs are between 35 and 42 nucleotides in length and are derived from centromeric elements containing a kangaroospecific retrovirus. Although the biogenesis and function of this new class of RNAs are unknown, their sequences are characterized by a mysterious and distinct motif.

\section{HOX genes and the need for speed}

What makes those legs? Although the forelimbs of the tammar are small, the hindlimbs are well-developed and muscular power units. This body plan is dictated by the HOX genes [7], which are a closely related group of transcription factors that play an important role in establishing the anteroposterior axis. The genome sequence of the tammar reveals that the tammar HOX genes are highly conserved with those of mouse and human, suggesting that differences in body plan may be the result of regulatory differences. Intriguingly, the authors speculate that regulation of HOXD13, which is strongly expressed in the anterior and posterior points of the hindlimbs, could direct morphological specialization allowing for the tammar's characteristic hop. Now that the genome of the tammar has been sequenced, the regulatory control of these $H O X$ genes can be dissected. This will doubtlessly provide fascinating insights into the formation of the tammar's body plan.

\section{Mummy roos}

Marsupials give birth to highly altricial young that complete most of their development in the pouch, attached to the mother's teat. Despite kangaroo mothers' attempts to clean out the pouch prior to the arrival of the newborn, the pouch is a dangerous place teeming with potentially deadly pathogens. External development requires marsupial young to be especially equipped to evade pathogenic attack. To make matters worse, the tiny pouch young do not even have mature immune systems. Ingeniously, wallabies protect their offspring by delivering secret weapons in the milk. The wallaby genome contains 14 cathelicidin genes, encoding a family of powerful antimicrobial peptides that are expressed in the mammary gland and secreted into the milk. Indeed, immune genes have undergone a significant reorganization in the wallaby genome, most notably with the dispersal of MHC genes onto several different chromosomes.

The marsupial lineage has undergone remarkable innovations in lactation. The composition of marsupial milk is perfectly matched with the developmental stage of the pouch young, and it is even possible for a single mother to dispense two types of milk to support young at different stages in their development. Perhaps unsurprisingly, the wallaby genome contains a large number of novel genes that are predicted to encode milk proteins. The tammar genome will be an essential tool for future work directed at understanding the composition and production of marsupial milk.

\section{Smell you later}

One disadvantage of being small, rather defenseless and tasty is that you become lunch. To stay one jump ahead of potential diners, the tammar has developed a highly tuned sense of smell. Remarkably, the genome and transcriptome sequence of the tammar identified as many as 1,500 olfactory receptor genes, providing the tammar with a remarkably large range of odor detection. These genes are likely to serve a variety of functions, including helping the newborn tammar to locate the teat 
within the pouch, find food and detect predators. The genome sequence of the tammar and the annotation of the olfactory genes will play an important role in helping us to understand the mechanisms of olfaction in the tammar.

The tammar genome sequence provides a fascinating window on the evolution of mammals and represents a critical resource for the community that will be mined for years to come.

\section{Abbreviations}

MHC, major histocompatibility complex; miRNA, microRNA; piRNA, piwi-interacting RNA.

\section{Competing interests}

The authors declare that they have no competing interests.

\section{Acknowledgements}

The authors wish to acknowledge Dan Keeffe for kindly providing his photograph for use as Figure 1

Published: 19 August 2011

\section{References}

1. Luo ZX: Transformation and diversification in early mammal evolution. Nature 2007, 450:1011-1019.
2. Bininda-Emonds OR, Cardillo M, Jones KE, MacPhee RD, Beck RM, Grenyer R, Price SA, Vos RA, Gittleman JL, Purvis A: The delayed rise of present-day mammals. Nature 2007, 446:507-512.

3. Tammar Wallaby Genome Sequencing Consortium: Genome sequence of an Australian kangaroo, Macropus eugenii, provides insight into the evolution of mammalian reproduction and development. Genome Bio/ 2011, 12: R81

4. Mikkelsen TS, Wakefield MJ, Aken B, Amemiya CT, Chang JL, Duke S, Garber M, Gentles AJ, Goodstadt L, Heger A, Jurka J, Kamal M, Mauceli E, Searle SM, Sharpe T, Baker ML, Batzer MA, Benos PV, Belov K, Clamp M, Cook A, Cuff J, Das R, Davidow L, Deakin JE, Fazzari MJ, Glass JL, Grabherr M, Greally JM, Gu W, et al:: Genome of the marsupial Monodelphis domestica reveals innovation in non-coding sequences. Nature 2007, 447:167-177.

5. Carone DM, Longo MS, Ferreri GC, Hall L, Harris M, Shook N, Bulazel KV, Carone BR, Obergfell C, O'Neill MJ, O'Neill RJ: A new class of retroviral and satellite encoded small RNAs emanates from mammalian centromeres. Chromosoma 2009, 118:113-125.

6. Brown CJ, Ballabio A, Rupert JL, Lafreniere RG, Grompe M, Tonlorenzi R, Willard HF: A gene from the region of the human $X$ inactivation centre is expressed exclusively from the inactive X chromosome. Nature 1991, 349:38-44.

7. Garcia-Fernandez J: The genesis and evolution of homeobox gene clusters. Nat Rev Genet 2005, 6:881-892.

doi:10.1186/gb-2011-12-8-123

Cite this article as: Murchison EP, Adams DJ: Sequencing Skippy: the genome sequence of an Australian kangaroo, Macropus eugenii. Genome Biology 2011, 12:123. 